\title{
Disease presentation and surgical treatment of patients with foreign-body granulomas and ASIA syndrome: case series
}

\author{
Javier Lopez-Mendoza ${ }^{1}$, Edgar Vargas-Flores ${ }^{2}$, Nicole Mouneu-Ornelas ${ }^{3}$, \\ Carlos Altamirano-Arcos ${ }^{2}$
}

${ }^{1}$ Department of Plastic, Aesthetic and Reconstructive Surgery, Hospital Angeles del Pedregal, Mexico City; ${ }^{2}$ Department of Plastic, Aesthetic and Reconstructive Surgery, Hospital General Dr. Manuel Gea Gonzalez, Mexico City; ${ }^{3}$ Department of Rheumatology, Hospital Angeles del Pedregal, Mexico City, Mexico

\begin{abstract}
Background The result of illicit polymer injection is chronic inflammation with foreign-body granuloma (FBG) formation. Treatment can be divided into medical and surgical. Some patients develop severe complications with need surgical treatment. This study aims to describe patients who underwent surgical removal of the FBGs and autoimmune/inflammatory syndrome induced by adjuvants (ASIA); additionally, we evaluated the quality of life after surgery. Methods In this retrospective single-center study, the authors examined data of patients who underwent surgical removal of FBG caused by illicit polymer injection for cosmetic purposes and confirmed ASIA from 2015 to 2020 by three different surgical approaches. Descriptive summary statistics were reported on patient demographics, presenting symptoms and clinical examination features, treatment strategies, histopathology reports and quality of life. Results The cohort included 11 female patients with FBGs and ASIA. The most affected anatomical zones were the combination of gluteal region, thighs and legs (40\%); and thighs with legs (20\%). Main presentation was: skin hyperpigmentation (90.9\%), skin induration (63.6\%), chronic fatigue $(63.6 \%)$, and ulcers (36.4\%). Surgical modalities consisted of: ultrasonic-assisted liposuction in four patients (36.4\%); open en bloc excision and primary closure in four patients (36.4\%); and open en bloc excision and microsurgical reconstruction in three patients (27.2\%). The postoperative quality of life visual analog scale score was 83.9.

Conclusions ASIA treatment represents a challenge for the plastic surgeon. Adequate surgical treatment emphasizing, when possible, the total or near-total resection of the FBG must be performed to improve ASIA evolution.
\end{abstract}

Keywords Quality of life / Granuloma, foreign-body / Polymers / Xenobiotics

pISSN: 2234-6163 • elSSN: 2234-6171 • https://doi.org/10.5999/aps.2020.02152 • Arch Plast Surg 2021;48:366-372

\author{
Correspondence: \\ Javier Lopez-Mendoza \\ Department of Plastic, Aesthetic and \\ Reconstructive Surgery, Hospital \\ Angeles del Pedregal, Camino a Santa \\ Teresa No. 1055, Col. Héroes de \\ Padierna, Consultorio 605, Mexico \\ City 10700, Mexico \\ Tel: +52-55-4088-2248 \\ Fax: +52-55-5135-4285 \\ E-mail: lopezmendozamd@gmail.com
}

\section{INTRODUCTION}

Human adjuvant disease was described in 1964 by Miyoshi et al. [1] as an immunological mediated disorder, caused by expo- sure to foreign substances that can act as adjuvants, and that are used for cosmetic purposes $[1,2]$. These substances include mainly: paraffin oil, mineral oil, and silicon [3]. The immediate satisfactory results on a cosmetic level, the low cost, and its easy

Copyright $\odot 2021$ The Korean Society of Plastic and Reconstructive Surgeons

This is an Open Access article distributed under the terms of the Creative Commons Attribution Non-Commercial License (https://creativecommons.org/

licenses/by-nc/4.0/) which permits unrestricted non-commercial use, distribution, and reproduction in any medium, provided the original work is properly cited. I www.e-aps.org 
access character have encouraged its rapid world spread [4].

Adjuvants are defined as immunological molecules that potentiate antigen-specific immune responses [5,6]. In 2011, Shoenfeld and Agmon-Levin [7] propose the term ASIA (autoimmune/inflammatory syndrome induced by adjuvants) syndrome, which encompasses immune-mediated conditions, all triggered by adjuvants and are the result of the interplay of genetic and environmental factors $[7,8]$.

Patients with ASIA syndrome present with a wide range of symptoms that can be mild or severe. Including fatigue, cognitive impairment, arthralgias, myalgias, pyrexia, dry eyes and dry mouth, calcification, abscesses, hair loss, chronic pain, among others, confirming the heterogeneity of this syndrome $[8,9]$. The impact on the quality of life, depends on the extent and the severity of lesions. Patients can have difficulties to walk or sit if the mineral oil was injected in the buttocks. Some are unable to work, which can lead to depression [6]. There is little information in the literature about depression and the deterioration of the quality of life in ASIA patients [10]. Most patients will require a psychological referral.

ASIA syndrome by mineral oil is defined as the infiltration of oily type modeling substances for cosmetic purposes [11]. The result of oil injection is chronic inflammation with granuloma formation [12]. There is thickening with the accumulation of collagen fibers oriented parallel to the superficial epithelium with an increase of fusiform fibroblasts at the dermis level [13].

An effective and long-term treatment has not been well established. Most of the time, it requires a multidisciplinary approach that should include plastic surgeons, rheumatologists, infectologists, dermatologists, and mental health professionals [5]. Treatment can be divided into medical and surgical treatment. There are no medications that can cure ASIA, but therapy can reduce symptoms [9]. The objective of surgical treatment is to remove most of the material to improve ASIA syndrome evolution [14]. The more substance is left, more relapses will appear and multiple secondary surgeries will be the rule; so, the complexity of the reconstructive procedure depends on the affected area, the dissemination of substances and the severity of the lesions.

This study aims to describe the disease presentation, patients' demographics and surgical planning of patients who underwent surgical excision of the foreign-body granulomas (FBG) and injured surrounding tissue with three different surgical approaches; consisted of: (1) ultrasonic-assisted liposuction (UAL), (2) open en bloc excision and primary closure, and (3) open en bloc excision and microsurgical reconstruction. Additionally, a postoperative evaluation of quality of life was made using the EuroQol-5ED tool (EQ-5D).

\section{METHODS}

\section{Patients}

We designed a single-center retrospective, consecutive case series study. Final cohort included 11 patients with the presence of FBG and ASIA syndrome because of illicit polymer injection and treated surgically with three different approaches from January 2015 to January 2020 all in a private practice setting and treated all by plastic surgeon senior author (JLM). Written informed consent was obtained from all patients for publication. This study conforms to the guiding principles of the Declaration of Helsinki. This study was registered in ClinicalTrials.gov (NCT04579744).

We reviewed the medical records, which included pre- and postoperative photographs of the region of adjuvants application and the notes of the findings during the operation.

UAL has been proposed for the treatment of siliconomas and FBG $[15,16]$. In our cases with a finger-pattern dissemination or not well-demarcated FBG, we used UAL as the first surgical option. Open approach was used if patients presented one or more Park parameters: (1) history of multiple unsuccessful nonsurgical therapies, (2) a well-demarcated and loculated chronic lesion with a diameter greater than $3 \mathrm{~cm}$, (3) severe infectious signs accompanying sensory dysfunction or tissue necrosis, and (4) newly developed psychological problems due to recognition of severe contour deformity [17].

The groups were divided accordingly: group 1, polymer disseminated to buttocks and/or lower extremities; group 2, FBG isolated to buttocks with moderate to severe skin changes, and group 3, FBG isolated to lower legs with moderate to severe skin changes. The extent and the volume of material in the affected area was relevant in the decision of treatment technique, for this reason, a preoperative magnetic resonance image was mandatory.

Patients were treated surgically with UAL for group 1 ; open en bloc excision and primary closure for group 2; and open en bloc excision and microsurgical reconstruction for group 3 (Figs. 1-3).

In order to evaluate the quality of life, we used the EQ-5D health assessment index value in all patients at their last followup visit. EQ-5D is a descriptive system that consists of five health domains (mobility, self-care, usual activities, pain/discomfort, and anxiety/depression). Each domain has five levels: no problems, slight problems, moderate problems, severe problems, and extreme problems and also includes a general health visual analog scale (VAS) from 0 (worst health imaginable) to 100 (best health imaginable).

Collected demographic and clinical data included patient's sex, age and educational level [18]. Preoperative clinical data comprised time with the injected substance, involved areas, presence of fever, chronic fatigue, skin hyperpigmentation, skin ulcers, 

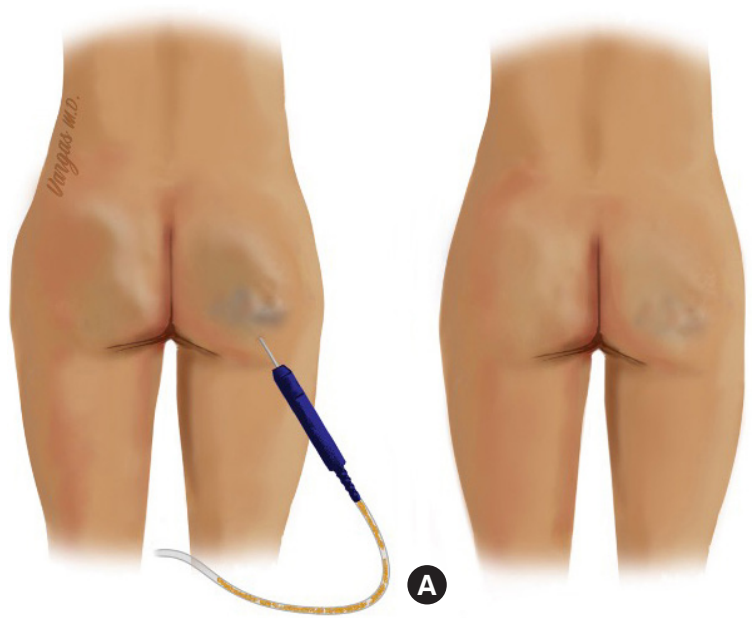

Fig. 1. Ultrasonic liposuction for biopolymer extraction. (A) Intraoperative appearance. (B) Postoperative result.
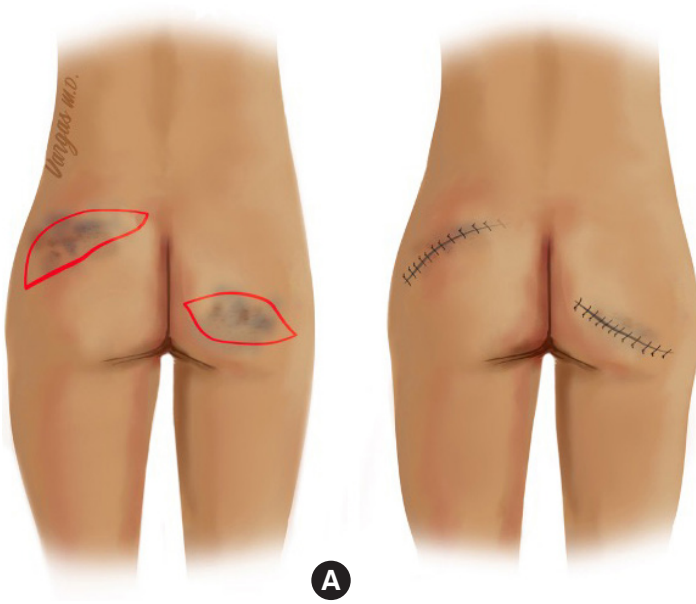

A

B

Fig. 2. (A) Preoperative appearance and resection markings. (B) Postoperative appearance after primary closure.
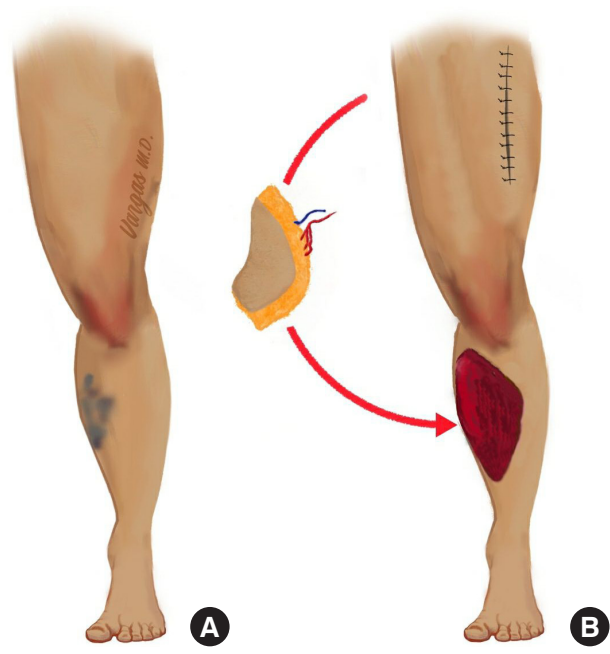

Fig. 3. Resection and microsurgical reconstruction. (A) Preoperative appearance with biopolymers in the leg. (B) Anterolateral thigh flap for reconstruction. previous medical treatment. Operative data encompassed surgical procedure and complications and finally, quality of life was assessed.

\section{Statistical analysis}

Descriptive analysis was performed using mean, standard deviation, and data range. Descriptive and analytic statistics results were obtained with the SPSS statistical analysis software version 25.0 (IBM Corp., Armonk, NY, USA).

\section{RESULTS}

\section{Patient population}

Eleven female patients with FBG and ASIA syndrome were included in this series. The average age of the patients was $49.3 \pm 10.3$ years (range, 29-62 years). Regarding the educational level, seven patients $(63.6 \%)$ completed high school, three (27.3\%) were professional and one (9.1\%) had a technical career (Table 1). All patients were injected by non-health related professionals; the foreign substance used was not specified by patients but refer it as: "collagen" or "simple oil." The mean follow-up was 31.1 months (range, 6-96 months). Concerning the preoperative clinical data: 10 patients (90.9\%) reported skin hyperpigmentation, seven $(63.6 \%)$ skin induration in the affected area, seven (63.6\%) chronic fatigue, four (36.4\%) skin ulcers, two (18.2\%) fever, and seven (63.6\%) received previous medical treatment (Table 2). The most affected anatomical zones were the combination of gluteal region + thighs + legs in five patients (45.5\%), followed by gluteal region + legs in two patients $(18.2 \%)$ and gluteal region alone in two patients (18.2\%) (Fig. 4).

\section{Surgical treatment and patient management}

Group 1 consisted of four patients (36.4\%), group 2 of four pa-

Table 1. Patient demographics

\begin{tabular}{lc}
\hline Characteristic & Value $(\mathrm{n}=11)$ \\
\hline Age (yr), mean \pm SD (range) & $49.3 \pm 10.3(29-62)$ \\
Sex, No. (\%) & 0 \\
Male & $11(100)$ \\
Female & \\
Educational level, No. (\%) & 0 \\
No education & 0 \\
Primary & 0 \\
Secondary & $7(63.6)$ \\
High school & $1(9.1)$ \\
Technical career & $3(27.3)$ \\
Bachelor's or professional & 0 \\
Postgraduate & $31.1(6-96)$ \\
Follow-up at EQ-5D (mo), mean (range)
\end{tabular}

EQ-5D, EuroQol-5ED tool. 
tients (36.4\%) (Fig. 5) and group 3 of three patients (27.2\%) (Fig. 6). All patients were managed as in-patients and discharged at 1st postoperative day in group 1 and 2; and 5th day in group 3. Main complications per group were, group 1 skin thermal burn due ultrasound; in group 2 and 3 were wound dehiscence. Complications were managed in outpatient department. In group 3, a flap survival rate of $100 \%$ was reported. For all groups no mortality was reported.

\section{Histopathology}

Five samples were analyzed, three from group 3 and two from group 2, and reported as: fat necrosis, xanthogranulomatous

Table 2. Preoperative clinical data

\begin{tabular}{lc}
\hline Variable & No. (\%) \\
\hline Skin stiffness & $7(63.6)$ \\
Yes & $4(36.4)$ \\
No & \\
Fever & $2(18.2)$ \\
Yes & $9(81.8)$ \\
No & \\
Chronic fatigue & $7(63.6)$ \\
Yes & $4(36.4)$ \\
No & \\
Hyperpigmentation & $10(90.9)$ \\
Yes & $1(9.1)$ \\
No & \\
Presence of ulcers & $4(36.4)$ \\
Yes & $7(63.6)$ \\
No & \\
Previous medical treatment & $7(63.6)$ \\
Yes & $4(36.4)$ \\
No
\end{tabular}

chronic inflammation with severe foreign-body reaction and fibrosis. Two patients also had reaction to oily material and coagulative necrosis (groups 2 and 3 ).

\section{Quality of life}

Moderate or severe concerns in the EQ-5D questionnaire were pain/discomfort (30\%) and anxiety/depression (20\%).

Regarding the quality of life VAS score, the average score of the population was $82.6 \pm 17$ points (range, $45-100$ ). Per group, in group 1 was $75 \pm 22.7$ points (range, 45-95), in group 2 was $79.7 \pm 11.8$ points (range, $70-94$ ), and in group 3 was $96.6 \pm 5.7$ points (range, 45-100); without any intergroup statistical significance $(\mathrm{P}=0.2)$.
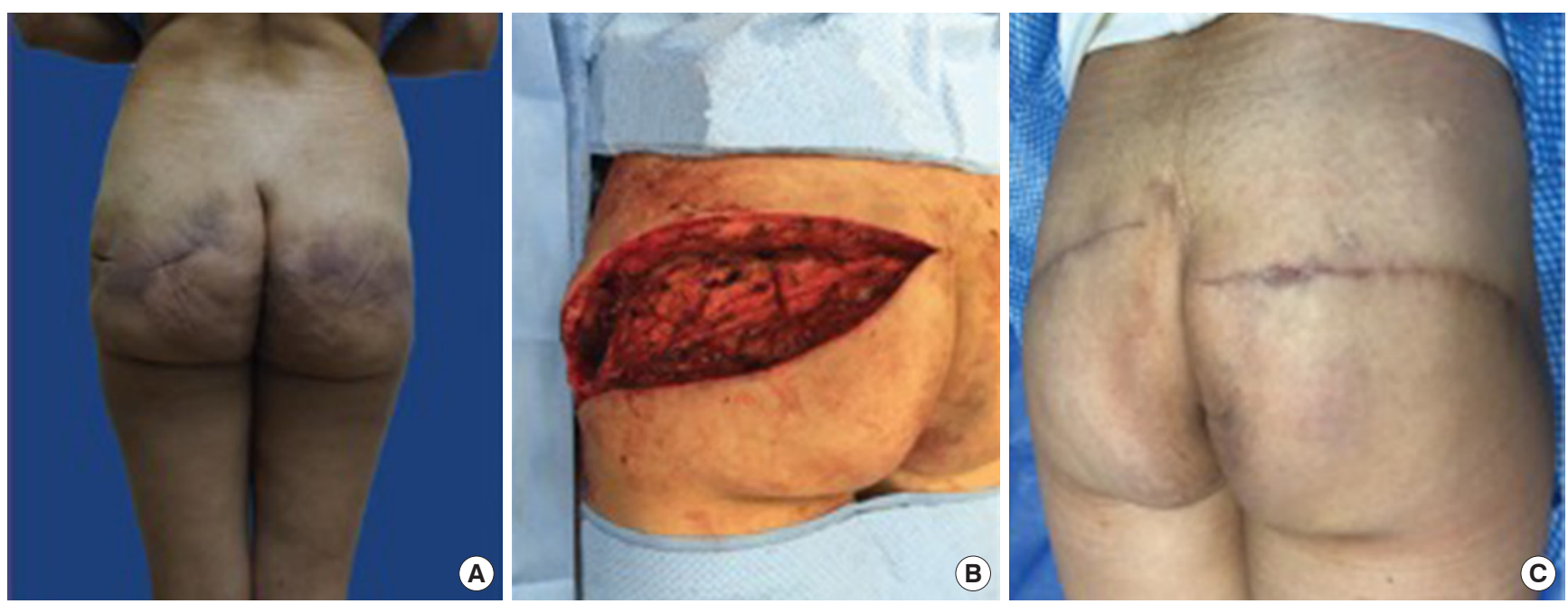

Fig. 5. A 45-year-old female patient with a 17-year history of biopolymers in the gluteal area surgically treated with en bloc excision and primary closure. (A) Preoperative view with hyperpigmented and atrophic skin areas, (B) intraoperative view with the area of excision, and (C) postoperative view 2 weeks after closure. 

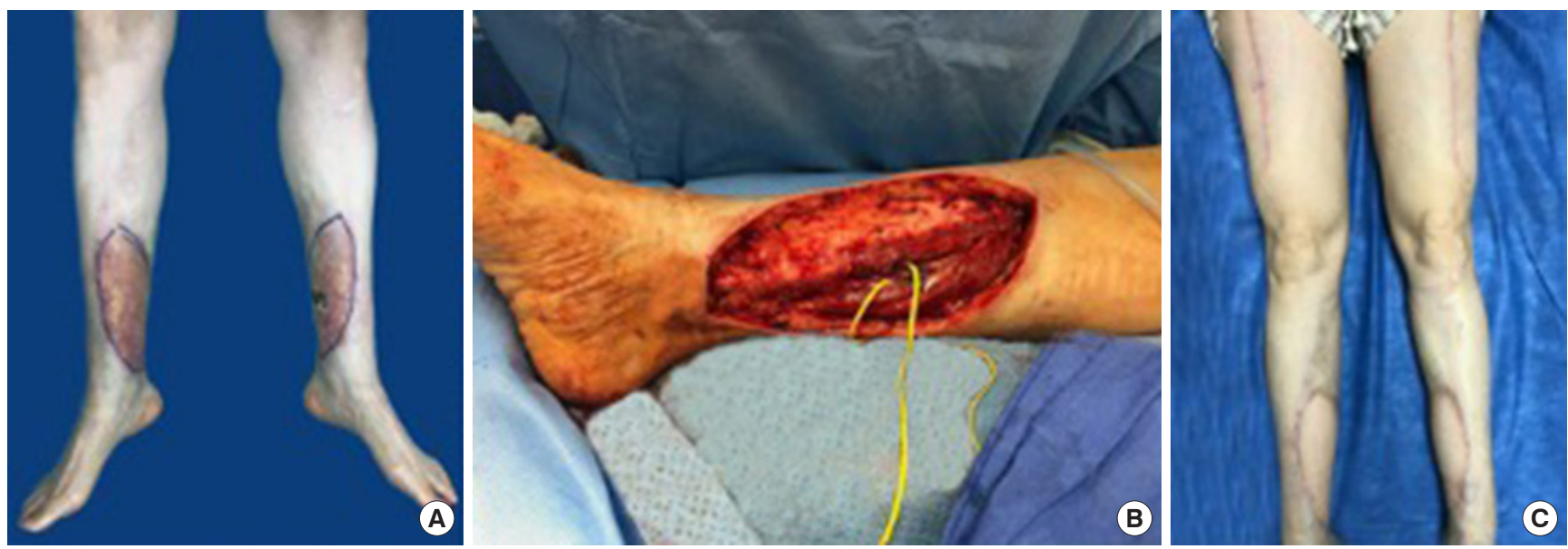

Fig. 6. A 48 -year-old female patient with a 12-year history of biopolymers in the legs surgically treated with en bloc excision and closure with bilateral anterolateral thigh flaps. (A) Preoperative, (B) intraoperative, and (C) postoperative views.

\section{DISCUSSION}

Despite the large number of reports showing the harmful effects of these substances, they are still indiscriminately used as a consequence of the growing demand for cosmetic procedures [3].

This pathology is seen mainly in women, as we can see in our results. However, there are reports about its use in transgender patients [19]. In our results, the less educated women were most affected; so probably this group has major risks because the lack of information at the moment to decide where to do an aesthetic procedure. In this series, the presentations of the leading symptoms were skin hyperpigmentation (90.9\%), chronic fatigue $(63.6 \%)$, skin induration $(63.6 \%)$, skin ulcers $(36.4 \%)$, and fever (18.2\%). This presentation was already described by many authors and described as an immune reaction to the foreign substance.

In the literature is described the use of medical treatment and laser to treat symptoms and diminish the immune reaction, 63\% of our patients received medical treatment and had minimal improvement of their symptoms; this leads them to seek surgical treatment as previous report suggested [17].

We performed a histopathologic study in five cases to identify the injected materials. However, microscopic examinations revealed only inflammatory foreign-body reactions without any information about the injected materials. Additionally, studies to assess the exact compound injected incurred in additional costs for the patients, and probably will not change the surgical method.

UAL has been proposed as a method to remove siliconomas; in this series, we performed this method in four patients (36.4\%). As Zandi [20] proposed, this technique works well in patients with small droplets of the injected viscous material, producing less bleeding and a short operative time [20]. Magnetic resonance imaging is extremely important to identify this in the preoperative period. Otherwise, open approach was proposed by Park et al. [17] due ineffectiveness of laser or liposuction. With an open approach we treated seven patients, identified with 1 or more Park criteria. The main criteria in this series was a well-demarcated or loculated chronic lesion with a diameter greater than $3 \mathrm{~cm}$. The open approach gave us the possibility to remove skin, FBG and injured tissues. Lee and Kim [21] stated that the skin has a very strong immune action against foreign substances; for this reason, removal of skin could have a major benefit comparing with liposuction alone. But this theory was not tested in this study.

Anxiety and depression are frequent reactions to unwanted complications FBG and ASIA: pain, tumors, deformities, skin problems, and chronic systemic repercussions [22]. However, there is no data linking fatigue syndromes, anxiety, or depression to the toxic substances themself. Vera-Lastra et al. [10] reported an incidence of depression in ASIA patients of $72 \%$. Overall quality of life after FBG excision in ASIA has not been previously reported. In our study, was of $82.6 \%$; with a prevalence of anxiety and pain of $54.6 \%$. Our incidence is lower than previous literature reports probably because partial or total removal of the foreign material improved ASIA symptoms and impacted directly on quality of life. Comparing our results with the population norms for the EQ-5D where females between 35 to 44 reported a VAS of 80; put our cohort in a similar quality of life of healthy females [23]. We identified a slight tendency of major scores in patients who underwent major excisions compared to UAL, but not relevant to make conclusions nor propose an efficient surgical method.

The reconstruction of defects arising after en bloc excision of 
the affected tissue, due to the infiltration of modeling substances, is becoming worldwide accepted, especially in a patient with severely impaired quality of life. Major skin disease will need major skin removal and further skin coverage as part of surgical treatment; but as we know, complete removal of foreign material is the gold standard to diminish the immune reaction against the adjuvant; and proper reconstruction guarantees stable coverage with less relapses of the disease, better control of the immunologic response, and an acceptable functional and aesthetic result [24].

This study has limitations; it has been designed as a retrospective, and the sample size is small. A prospective study with a larger population and with biomarkers assessment is needed.

In conclusion, ASIA syndrome and FBG treatment represents a challenge for the plastic surgeon, because excision of the infiltrated material is usually complex and with severe aesthetic sequels. Moreover, complete resection cannot often be performed, frustrating both the patient and the surgeon. Adequate surgical treatment emphasizing, when possible, the total or near-total resection of the substance must be performed. Major excisions of the affected tissue and stable wound coverage could lead to longer symptom-free periods. Our next goal will be, compare preoperative and postoperative changes in quality of life to determine the maximal benefit of each surgical procedure; also, an adequate investigation of materials injected should be done to assess the potential damage of each substance to the tissues.

\section{NOTES}

\section{Conflict of interest}

No potential conflict of interest relevant to this article was reported.

\section{Ethical approval}

The study was approved by the Institutional Review Board of AMCPER/HAP (IRB No. AMCPER 10-2020) and performed in accordance with the principles of the Declaration of Helsinki. Written informed consent was obtained.

\section{Patient consent}

The patients provided written informed consent for the publication and the use of their images.

\section{Author contribution}

Conceptualization: J Lopez-Mendoza, N Mouneu-Ornelas. Data curation: N Mouneu-Ornelas, C Altamirano-Arcos. Formal analysis: C Altamirano-Arcos. Methodology: J Lopez-Mendoza. Project administration: J Lopez-Mendoza, N Mouneu-Ornelas.
Visualization: J Lopez-Mendoza, E Vargas-Flores. Writing - original draft: J Lopez-Mendoza, E Vargas-Flores, N Mouneu-Ornelas, C Altamirano-Arcos. Writing - review \& editing: J LopezMendoza.

\section{ORCID}

Javier Lopez-Mendoza https://orcid.org/0000-0001-9065-198X Edgar Vargas-Flores https://orcid.org/0000-0002-0430-8841 Nicole Mouneu-Ornelas

https://orcid.org/0000-0003-2884-9439

Carlos Altamirano-Arcos

$$
\text { https://orcid.org/0000-0002-8549-7073 }
$$

\section{REFERENCES}

1. Miyoshi K, Miyamura T, Kobayashi T, et al. Hypergammaglobulinemia by prolonged adjuvanticity in men: disorders developed after augmentation mammoplasty. J Keio Med Soc 1964;2122:9-14.

2. Baldwin CM Jr, Kaplan EN. Silicone-induced human adjuvant disease? Ann Plast Surg 1983;10:270-3.

3. Cardenas-Camarena L. Managing the mammary gland infiltrated with foreign substances: different surgical alternatives. Ann Plast Surg 2009;62:621-6.

4. Ahmed U, Freeman A, Kirkham A, et al. Self injection of foreign materials into the penis. Ann R Coll Surg Engl 2017; 99:e78-82.

5. Martinez-Villarreal AA, Asz-Sigall D, Gutierrez-Mendoza D, et al. A case series and a review of the literature on foreign modelling agent reaction: an emerging problem. Int Wound J 2017;14:546-54.

6. Vera-Lastra O, Medina G, Cruz-Dominguez Mdel P, et al. Human adjuvant disease induced by foreign substances: a new model of ASIA (Shoenfeld's syndrome). Lupus 2012; 21:128-35.

7. Shoenfeld Y, Agmon-Levin N. 'ASIA': autoimmune/inflammatory syndrome induced by adjuvants. J Autoimmun 2011; 36:4-8.

8. Vera-Lastra O, Medina G, Cruz-Dominguez MP, et al. Autoimmune/inflammatory syndrome induced by mineral oil: a health problem. Clin Rheumatol 2018;37:1441-8.

9. Cohen Tervaert JW. Autoinflammatory/autoimmunity syndrome induced by adjuvants (ASIA; Shoenfeld's syndrome): a new flame. Autoimmun Rev 2018;17:1259-64.

10. Vera-Lastra O, Hernadez PB, Sanchez-Rodriguez A, et al. AB0991 Prevalence of fibromyalgia and depression in patients with autoimmune/inflammatory syndrome induced 
by adjuvants compared to patients with systemic sclerosis. Ann Rheum Dis 2017;76(Suppl 2):1401.

11. Petry T, Bury D, Fautz R, et al. Review of data on the dermal penetration of mineral oils and waxes used in cosmetic applications. Toxicol Lett 2017;280:70-8.

12. Vera-Lastra O, Medina G, Cruz-Dominguez Mdel P, et al. Autoimmune/inflammatory syndrome induced by adjuvants (Shoenfeld's syndrome): clinical and immunological spectrum. Expert Rev Clin Immunol 2013;9:361-73.

13. Alcocer-Varela J, Alarcon-Segovia D. Decreased production of and response to interleukin-2 by cultured lymphocytes from patients with systemic lupus erythematosus. J Clin Invest 1982;69:1388-92.

14. Dagan A, Kogan M, Shoenfeld Y, et al. When uncommon and common coalesce: adult onset Still's disease associated with breast augmentation as part of autoimmune syndrome induced by adjuvants (ASIA). Clin Rheumatol 2016;35:16438.

15. Grippaudo FR, Spalvieri C, Rossi A, et al. Ultrasound-assisted liposuction for the removal of siliconomas. Scand J Plast Reconstr Surg Hand Surg 2004;38:21-6.

16. Bassetto F, Abatangelo S, Masetto L, et al. Ultrasound-assisted liposuction as a safe and effective method for the removal of siliconomas. Aesthetic Plast Surg 2012;36:220-2.

17. Park TH, Seo SW, Kim JK, et al. Clinical outcome in a series of 173 cases of foreign body granuloma: improved out- comes with a novel surgical technique. J Plast Reconstr Aesthet Surg 2012;65:29-34.

18. EuroQol Group. EuroQol: a new facility for the measurement of health-related quality of life. Health Policy 1990;16: 199-208.

19. Colaris MJL, de Boer M, van der Hulst RR, et al. Two hundreds cases of ASIA syndrome following silicone implants: a comparative study of 30 years and a review of current literature. Immunol Res 2017;65:120-8.

20. Zandi I. Removal of soft tissue injected with liquid silicone with an ultrasound-suction system. Plast Reconstr Surg 2001; 107:1077.

21. Lee JM, Kim YJ. Foreign body granulomas after the use of dermal fillers: pathophysiology, clinical appearance, histologic features, and treatment. Arch Plast Surg 2015;42:2329.

22. Schenone GE. Bioethical considerations. In: Schenone G, editor. Injection-induced breast siliconomas. Cham: Springer; 2020. p. 271-88.

23. Janssen B, Szende A. Population Norms for the EQ-5D. In: Szende A, Janssen B, Cabases J, editors. Self-reported population health: an international perspective based on EQ-5D. Dordrecht: Springer; 2014. p. 19-30.

24. Mendoza JL, Tarriba JT, Reyes AI. Microsurgical management of severe human adjuvant disease due to mineral oil injection. Int Microsurg J 2020;4:1. 